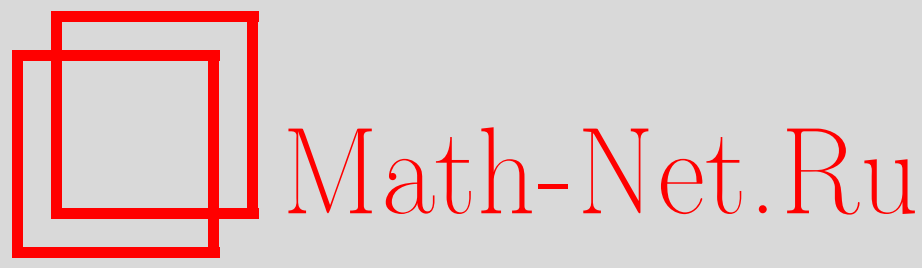

Д. В. Артамонов, Система Шлезингера и изомонодромные деформации расслоений со связностями на римановых поверхностях, ТМФ, 2012, том 171, номер 3, 370-386

DOI: https://doi.org/10.4213/tmf6899

Использование Общероссийского математического портала Math-Net.Ru подразумевает, что вы прочитали и согласны с пользовательским соглашением http://www . mathnet.ru/rus/agreement

Параметры загрузки:

IP : 34.227 .88 .159

26 апреля 2023 г., 13:35:13

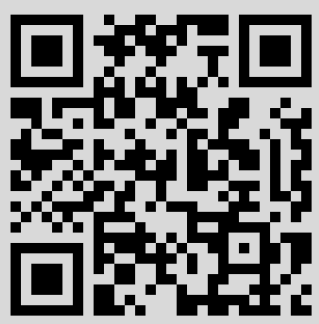




\title{
ФИЗИКА
}

Том 171, № 3

июнь, 2012

(C) 2012 г.

Д. В. Артамонов*

\section{СИСТЕМА ШЛЕЗИНГЕРА И ИЗОМОНОДРОМНЫЕ ДЕФОРМАЦИИ РАССЛОЕНИЙ СО СВЯЗНОСТЯМИ НА РИМАНОВЫХ ПОВЕРХНОСТЯХ}

\begin{abstract}
Вводится способ представления пар $(E, \nabla)$, где $E$ - расслоение на римановой поверхности, $\nabla$ - фуксова связность в $E$, который основан на представлении поверхности как фактора внешности единичного диска. В этом представлении выводятся локальные условия изомонодромности деформаций пар $(E, \nabla)$. Эти условия записываются как модифицированная система Шлезингера на сфере Римана (в типичном случае сводящаяся к обычной системе Шлезингера), дополненная некоторой системой линейных уравнений.
\end{abstract}

Ключевые слова: изомонодромные деформации, римановы поверхности, система Шлезингера.

\section{1. ВВЕДЕНИЕ}

Пусть задана система фуксовых дифференциальных уравнений на сфере Римана:

$$
\frac{d y}{d z}=\sum_{i} \frac{B_{i}}{z-a_{i}} y \text {. }
$$

Будем менять положение особых точек $a_{i}$ так, чтобы монодромия сохранялась и особые точки не сливались. Тогда вычеты $B_{i}$ превращаются в (многозначные) функции от $a_{i}$, а условие изомонодромности семейства систем

$$
\frac{d y}{d z}=\sum_{i} \frac{B_{i}\left(a_{1}, \ldots, a_{n}\right)}{z-a_{i}} y
$$

представляет собой некоторую систему нелинейных дифференциальных уравнений относительно функций $B_{i}$. В частности, если мы ограничимся шлезингеровскими деформациями (отметим, что в типичном случае все деформации являются шлезингеровскими; см., например, монографию [1]), то условие изомонодромности есть система Шлезингера.

${ }^{*}$ Московский государственный университет им. М. В. Ломоносова, Москва, Россия. E-mail: artamonov.dmitri@gmail.com 
Возьмем вместо сферы Римана риманову поверхность положительного рода. В этом случае естественно рассматривать деформации не систем линейных уравнений (другими словами, связностей в тривиальном расслоении), а изомонодромные деформации расслоений со связностями. При этом меняется как связность, так и расслоение. Аргументы в пользу того что необходимо менять расслоение, можно найти, например, во введении к работе [2]. Также естественно допускать деформацию модуля комплексной структуры. Подобные деформации рассматривались различными авторами в работах [3]-[9] и во многих других работах.

Как правило, рассматриваются частные случаи, например поверхности рода 1. Кричевер выписал уравнения, описывающие изомонодромные деформации в общем случае [5]. Его подход основан на мероморфной тривиальности расслоений над римановыми поверхностями, однако уравнения, полученные в работе [5], сильно отличаются от системы Шлезингера.

В случае рода 1 известен другой подход к описанию изомонодромных деформаций: эллиптическая система Шлезингера (см., например, работы [2], [10]), которая представляет собой систему уравнений, задающую изомонодромные деформации расслоений со связностями на торе; эта система является обобщением системы Шлезингера. В работе [2] высказано пожелание обобщить данную конструкцию на случай высших родов и выписать систему Шлезингера, что и будет сделано в настоящей работе. А именно, мы доказываем, что в случае римановых поверхностей изомонодромные деформации можно описать системой Шлезингера с добавлением некоторой системы линейных уравнений.

Приведем план работы. В разделе 2 описано пространство параметров деформаций: пространство Тейхмюллера с отмеченными точками - положениями особенностей связности. Мы берем именно пространство Тейхмюллера, а не пространство модулей комплексных структур по следующей причине: чтобы говорить о матрицах монодромии обхода по разрезам, нужно каким-либо образом зафиксировать эти разрезы, но пары "комплексная структура+система канонических разрезов" с точностью до отношения эквивалентности как раз образуют пространство Тейхмюллера. Для явного описания пространства параметров мы представляем риманову поверхность как фактор внешности единичного диска по действию группы Фукса. После этого каноническим способом выбирается фундаментальный $4 g$-угольник этого действия $(g-$ род поверхности). Особые точки представляются как точки на фундаментальном многоугольнике.

В разделе 3 предложен способ задания расслоений со связностями на римановой поверхности. Они описываются следующими данными: формой $\omega$ на сфере Римана и набором невырожденных матриц $S_{x_{0}^{1}, x_{0}^{i}}, i=2, \ldots, 4 g$, где индексы $x_{0}^{i}$ отвечают вершинам фундаментального многоугольника. Форма $\omega$ строится следующим образом. Существует отображение факторизации из фундаментального многоугольника на риманову поверхность. С его помощью мы берем результат обратного отображения расслоения со связностью на римановой поверхности на фундаментальный многоугольник. Полученное расслоение со связностью продолжается на всю сферу Римана с одной дополнительной особенностью в нуле. Далее мы фиксируем мероморфную тривиализацию этого расслоения на сфере Римана, являющуюся голоморофной тривиализацией на $\mathbb{C} \backslash\{0\}$. В указанной тривиализации связность в данном расслоении определяется некоторой формой, которая и есть форма $\omega$ (см. п. 3.1). 
Поясним, как возникают матрицы $S_{x_{0}^{1}}, x_{0}^{i}$. Расслоение со связностью на фундаментальном многоугольнике есть обратный образ расслоения со связностью на римановой поверхности. Все вершины многоугольника склеиваются в одну точку. Следовательно, существует оператор, отождествляющий слои над вершинами $x_{0}^{1}$ и $x_{0}^{i}$. Матрица $S_{x_{0}^{1}, x_{0}^{i}}$ есть матрица этого оператора (см. п. 3.2).

Указанных данных достаточно для восстановления расслоения со связностью на поверхности (см. теорему 1). Отметим, что процедура построения формы и матриц является неканонической: разные формы и матрицы могут давать эквивалентные расслоения. Однако всевозможные расслоения на поверхности не образуют "хорошего" пространства (см. обсуждение в работе [11]), поэтому не существует способа описания именно расслоений со связностями. По сути дела мы рассматриваем деформацию не расслоений со связностями, а данных, введенных выше (в работе [5] вместо расслоений со связностями фактически рассмотрены параметры мероморфной тривиализации, называемые параметрами Тюрина).

В разделе 4 определены шлезингеровские деформации расслоения со связностью на римановой поверхности и получены уравнения, описывающие изменение данных, введенных в разделе 3, при шлезингеровских изомонодромных деформациях. Доказано, что изомонодромные деформации описываются системой нелинейных уравнений относительно коэффициентов формы $\omega$ (в типичном случае - просто системой Шлезингера) и некоторой линейной системой для матриц $S_{x_{0}^{1} x_{0}^{i}}$ (см. теорему 2). Уравнения Шлезингера можно представить как интегрируемую гамильтонову систему. Таким образом, в рамках описываемого подхода к изомонодромным деформациям переход к положительному роду не приводит к появлению новых интегрируемых систем.

Подход настоящей работы и подход Кричевера [5] сопоставляются в разделе 5.

\section{2. ПРОСТРАНСТВО ПАРАМЕТРОВ ДЕФОРМАЦИЙ И ДЕФОРМИРУЕМЫЕ ОБЪЕКТЫ}

2.1. Пространство параметров. Пусть $M$ - риманова поверхность рода $g>1$, случай родов $g=0, g=1$ мы считаем тривиальным. Целью настоящей статьи является нахождение аналогов уравнений Шлезингера (обычных и эллиптических), известных для родов $g=0, g=1$, в случае высших родов.

Зафиксируем некоторую начальную точку $x_{0}$ на $M$.

ОПРедЕЛЕНиЕ 1 . Пусть $T$ есть пространство Тейхмюллера с $n$ отмеченными точками $a_{1}, \ldots, a_{n}$, где $a_{i} \neq a_{j}$ для $i \neq j$. Пространство $\widetilde{T}$ параметров деформации есть универсальное накрытие пространства $T$.

Возьмем образ точки $\tau \in \widetilde{T}$ в пространстве $T$. После этого можно говорить об отмеченных точках, отвечающих точке $\tau$, а также о комплексной структуре с системой канонических разрезов, отвечающей точке $\tau$, другими словами, о точке обычного пространства Тейхмюллера (без отмеченных точек), отвечающей точке $\tau$. Далее пространством Тейхмюллера мы будем называть обычное пространство Тейхмюллера без отмеченных точек, а его точки иногда будем называть отмеченными римановыми поверхностями.

Пространство $\widetilde{T}_{1}$, на котором определены изомонодромные семейства пар "расслоение+связность", строится следующим образом. 
ОПРЕДЕЛЕНИЕ 2. Пусть $T_{1}$ есть пространство Тейхмюллера с $n+1$ отмеченными точками $z, a_{1}, \ldots, a_{n}$, где $a_{i} \neq a_{j}$ при $i \neq j$. Тогда пространство $\widetilde{T}_{1}$ есть универсальное накрытие пространства $T_{1}$ по переменным $a_{i}$.

Существует отображение $\widetilde{T}_{1} \rightarrow \widetilde{T}$, “забывающее” отмеченную точку $z$.

ОПРЕДЕЛЕНИЕ 3 . Пусть $\tau \in \widetilde{T}$. Обозначим через $\left.\widetilde{T}_{1}\right|_{\tau}$ прообраз точки $\tau$ при отображении $\widetilde{T}_{1} \rightarrow \widetilde{T}$.

Пространство $\left.\widetilde{T}_{1}\right|_{\tau}$ следует понимать как риманову поверхность с комплексной структурой, каноническими разрезами и отмеченными точками $a_{1}, \ldots, a_{n}$, определяемыми точкой $\tau \in \widetilde{T}$.

Универсальное накрытие берется для того, чтобы обеспечить глобальное существование шлезингеровских деформаций при любом начальном условии (см. предложение 5 в разделе 4). В настоящей работе мы выпишем лишь локальные уравнения изомонодромных деформаций. Если рассматривать небольшие изменения параметров, то можно использовать $T$ вместо $\widetilde{T}$ как пространство параметров, при этом параметрами фактически являются просто положения особых точек и точка пространства Тейхмюллера. Деформируемый объект - это пара "расслоение+связность", а не форма (система линейных уравнений или, эквивалентно, связность в тривиальном расслоении, как в случае рода 0). При изменении положения особых точек изменяются и расслоение, и связность. Эволюция расслоения и связности однозначно определяется изменением точки пространства Тейхмюллера и положения особых точек.

2.2. Фундаментальный многоугольник. Пусть $D$ - внешность единичного диска. Точка пространства Тейхмюллера может быть задана набором автоморфизмов $Q_{1}, \ldots, Q_{2 g} \in$ Aut $D$, удовлетворяющих следующим свойствам [12]:

1) имеет место равенство $\prod_{j} Q_{2 j} Q_{2 j-1} Q_{2 j}^{-1} Q_{2 j-1}^{-1}=1$;

2 ) подгруппа $G \subset$ Aut $D$, порожденная $Q_{1}, \ldots, Q_{2 g}$, является группой Фукса;

3) существует фундаментальная область действия подгруппы $G \subset$ Aut $D$ на $D$, которая не пересекается с границей $\partial D$.

Поверхность $M$ восстанавливается как фактор внешности единичного диска по действию подгруппы $G \subset$ Aut $D$, порожденной автоморфизмами $Q_{1}, \ldots, Q_{2 g}$. Это действие имеет фундаментальную область, которая представляет собой неевклидов многоугольник $U$ с $4 g$ ребрами (неевклидовость означает, что каждое ребро есть неевклидова прямая). Канонические разрезы восстанавливаются как образы ребер фундаментального многоугольника.

Два набора $Q_{1}, \ldots, Q_{2 g}$ и $K_{1}, \ldots, K_{2 g}$ определяют одну и ту же точку пространства Тейхмюллера тогда и только тогда, когда существует автоморфизм $Q$ такой, что $K_{1}=Q Q_{1} Q^{-1}, \ldots, K_{2 g}=Q Q_{2 g} Q^{-1}$. Существует нормированный способ выбора системы автоморфизмов $Q_{1}, \ldots, Q_{2 g}$, при этом нормированная система восстанавливается по точке пространства Тейхмюллера однозначно. Традиционный способ нормировки описан в книге [12]. После фиксации нормировки можно построить канонический фундаментальный многоугольник, его вершины $x_{0}^{i}$ гладко (но не комплексно-аналитически) зависят от точки пространства Тейхмюллера.

Изменим традиционное определение нормированной системы образующих так, чтобы $x_{0}^{1} \equiv \infty$ независимо от точки пространства Тейхмюллера. Для этого выберем канонически $Q_{z}$ - автоморфизм множества $D$, переводящий $z$ в $\infty \in D$. Пусть 
$Q_{1}, \ldots, Q_{2 g}$ - нормированная в традиционном смысле система образующих, а $x_{0}^{1}-$ координаты вершин выбранного ранее фундаментального многоугольника $U$. Возьмем новые образующие $Q_{x_{0}^{1}} Q_{1} Q_{x_{0}^{1}}^{-1}, \ldots, Q_{x_{0}^{1}} Q_{2 g} Q_{x_{0}^{1}}^{-1}$. Они определяют ту же точку в пространстве Тейхмюллера. Кроме того, $Q_{x_{0}^{1}}(U)$ является фундаментальным многоугольником для действия группы Фукса, заданной новыми образующими. У многоугольника $Q_{x_{0}^{1}}(U)$ первая вершина есть $\infty$.

Подведем итог.

ПРЕДЛОЖЕНИЕ 1. Существует способ канонически выбрать фундаменталъный многоугольник для действия так, что он (т.е. координаты его вершин) гладко (но не комплексно-аналитически) зависит от точки пространства Тейхмюллера, а одна из вериин есть бесконечность.

Данный многоугольник далее мы обозначаем как $U$, а его вершины - как $x_{0}^{i}$, $i=1, \ldots, 4 g$, при этом $x_{0}^{1}=\infty$. Отмеченные точки $\left(z, a_{1}, \ldots, a_{n}\right)$ или $\left(a_{1}, \ldots, a_{n}\right)$ становятся точками многоугольника $U$.

При изучении деформаций в разделе 4 мы запрещаем переход особенностей связности через канонические разрезы, что не ограничивает общность, так как мы изучаем только локальные деформации.

\section{3. ОПИСАНИЕ РАССЛОЕНИЙ СО СВЯЗНОСТЯМИ НА РИМАНОВОЙ ПОВЕРХНОСТИ}

Пусть $E$ - расслоение на поверхности $M$, а $\nabla$ - связность в $E$ с особенностями в точках $a_{1}, \ldots, a_{n} \in M$. Предположим, что начальная точка $x_{0} \in M$ неособая. В настоящем разделе мы строим по паре $(E, \nabla)$ на римановой поверхности форму на сфере Римана и некоторые матрицы. Исходя из этих данных можно восстановить пару $(E, \nabla)$ на поверхности. Отметим, что построение формы на сфере является неканоническим, так как на определенном этапе рассуждений фиксируется тривиализация некоторого расслоения.

3.1. Построение формы на сфере Римана. Прежде всего построим форму на фундаментальном многоугольнике. Выше мы представили отмеченную риманову поверхность как фактор фундаментального многоугольника $U$. Пусть $\left(E_{U}, \nabla_{U}\right)-$ расслоение и связность на $U$, полученные как обратный образ пары $(E, \nabla)$ при факторизации. Продолжим пару $\left(E_{U}, \nabla_{U}\right)$ до пары $\left(E_{\overline{\mathbb{C}}}, \nabla_{\overline{\mathbb{C}}}\right)$ на всей сфере Римана. Для этого посмотрим, какую монодромию имеет связность $\nabla_{U}$ при обходе по пути $\gamma=\partial U$. Легко увидеть, что этому обходу соответствует монодромия $M_{\gamma}=M_{a_{1}} \ldots M_{a_{n}}$. Здесь монодромия $\nabla$ обхода по пути $\gamma$ обозначена через $M_{\gamma}$, а монодромия обхода вокруг особой точки $a_{i}$ - через $M_{a_{i}}, i=1, \ldots, n$.

Возьмем в области $\overline{\mathbb{C}} \backslash U$ тривиальное расслоение $E^{\prime}$. В качестве связности $\nabla^{\prime}$ возьмем связность с единственной особой точкой в нуле и монодромией $M_{\gamma}$ обхода вокруг нуля. Таким образом, на границе $\partial U$ расслоения $E_{U}$ и $E^{\prime}$ тривиальны и связности $\nabla_{U}$ и $\nabla^{\prime}$ в них имеют одинаковую монодромию. Из этого следует, что мы можем склеить пары $\left(E_{U}, \nabla_{U}\right)$ и $\left(E^{\prime}, \nabla^{\prime}\right)$ в пару $\left(E_{\overline{\mathbb{C}}}, \nabla_{\overline{\mathbb{C}}}\right)$ над всей сферой Римана. Для этого нужно склеить их по горизонтальным сечениям над границей $\partial U$. Опишем процедуру склейки по горизонтальным сечениям подробно, так она будет использоваться неоднократно. 
ПреДЛОЖЕНИЕ 2. Пусть $V$ - область на римановой поверхности, $\gamma$ - незамкнутая кривая без самопересечения в этой области, разбивающая ее на две части $V^{\prime}$ u $V^{\prime \prime}$. Существуют пары $\left(E^{\prime}, \nabla^{\prime}\right)$ на $V^{\prime} u\left(E^{\prime \prime}, \nabla^{\prime \prime}\right)$ на $V^{\prime \prime}$ без особенностей на $\gamma$. Пусть выбрано отождествление слоев $E_{P}^{\prime}=E_{P}^{\prime \prime}$ для некоторой точки $P \in \gamma$. Тогда существует однозначная прочедура склейки пар $\left(E^{\prime}, \nabla^{\prime}\right)$ u $\left(E^{\prime \prime}, \nabla^{\prime \prime}\right)$ в пару $(E, \nabla)$ на $V$. Если кривая замкнута, то процедура склейки осуществима тогда и только тогда, когда монодромии $\nabla^{\prime}$ и $\nabla^{\prime \prime}$ вдоль $\gamma$ совпадают.

ДокАзАТЕЛЬСтво данного утверждения известно, и мы его опускаем.

Любое расслоение на сфере Римана мероморфно тривиально. Более того, имеется мероморфная тривиализация, голоморфная на $\overline{\mathbb{C}} \backslash\{0\}$. Зафиксируем эту тривиализацию. При этом сечения станут голоморфными вектор-столбцами. Связность $\nabla_{\mathbb{C}}$ можно задать с помощью формы

$$
\omega=\left(\frac{C_{k}}{z^{k}}+\cdots+\frac{C_{1}}{z}+\sum_{i} \frac{B_{i}}{z-a_{i}}\right) d z
$$

с регулярной особенностью в нуле.

ОПРЕДЕЛЕНИЕ 4 . Пусть $\left(E_{U}, \nabla_{U}\right)$ есть обратный образ пары $(E, \nabla)$ при факторизации $U \rightarrow M$. Тогда $\omega$ есть форма связности $\nabla_{U}$ в такой тривиализации $E_{U}$, в которой она имеет вид (1) с регулярной особенностью в нуле.

Отметим, что указанная тривиализация неединственна.

ЗАмЕчАНИЕ 1. Для типичных матриц монодромии и типичных положений особых точек можно добиться того, что форма запишется просто как

$$
\omega=\left(\frac{C_{1}}{z}+\sum_{i=1}^{n} \frac{B_{i}}{z-a_{i}}\right) d z .
$$

В рассматриваемой нами ситуации $C_{1}=-\sum_{i=1}^{n} B_{i}$. Если мы положим $a_{0}=0$ и $B_{0}=-\sum_{i=1}^{n} B_{i}$, то

$$
\omega=\sum_{i=0}^{n} \frac{B_{i}}{z-a_{i}} d z
$$

3.2. Построение матриц операторов склейки $S_{x_{0}^{1}, x_{0}^{i}}$. Введем дополнительные объекты, с помощью которых по форме $\omega$ можно будет восстановить пару $(E, \nabla)$ над римановой поверхностью. Для восстановления расслоения над римановой поверхностью нам потребуются операторы склейки слоев $S_{z, z^{\prime}}: E_{U, z} \mapsto E_{U, z^{\prime}}$ из слоя расслоения $E_{U}$ над точкой $z$ в слой расслоения $E_{U}$ над точкой $z^{\prime}$. Эти операторы следующим образом определены для упорядоченной пары точек $z, z^{\prime} \in \partial U$, склеиваемых при факторизации $U \rightarrow M$.

ОПРЕДЕЛЕНИЕ 5. Расслоение $E_{U}$ есть обратный образ при факторизации расслоения $E$ на $M$, поэтому существуют изоморфизмы слоев $E_{U z} \rightarrow E_{Z}$ и $E_{U z^{\prime}} \rightarrow E_{Z}$. Определим $S_{z, z^{\prime}}$ как $E_{U, z} \rightarrow E_{Z} \rightarrow E_{U, z^{\prime}}$, где второе отображение есть обратное к $E_{U, z^{\prime}} \rightarrow E_{Z}$. 
Однако задание всех операторов $S_{z, z^{\prime}}$ избыточно. Пусть $x_{0}^{1}, \ldots, x_{0}^{4 g}-$ вершины фундаментального многоугольника. Ниже будет показано, что достаточно знать только операторы $S_{x_{0}^{1}, x_{0}^{i}}$. Так как тривиализация расслоения $E_{U}$ фиксирована, в дальнейшем мы будем говорить о матрицах $S_{x_{0}^{1}, x_{0}^{i}}$.

ОПРЕДЕЛЕНиЕ 6 . Матрицы $S_{x_{0}^{i}, x_{0}^{j}}$ есть матрицы операторов, осуществляющих склейку слоев над точками $x_{0}^{i}, x_{0}^{j}$ в смысле определения 5 .

Таким образом, расслоению со связностью на римановой поверхности $M$ и начальной точкой $x_{0}$ мы сопоставили форму $\omega$ вида (1) с особенностями $a_{i} \in U, i=1, \ldots, n$, и регулярной особенностью в нуле, а также набор матриц $S_{x_{0}^{1}, x_{0}^{i}}, i=1, \ldots, 4 g$.

3.3. Восстановление расслоения со связностью из формы $\omega$ и матриц $S_{x_{0}^{1}, x_{0}^{i}}$. Сначала мы рассмотрим процедуру восстановления пары на римановой поверхности в предположении, что форма на сфере и матрицы получены из некоторой пары на римановой поверхности, а затем исследуем вопрос, при каких форме и матрицах эта процедура осуществима.

Прежде всего построим пару $\left(E_{U}, \nabla_{U}\right)$ на фундаментальном многоугольнике: расслоение $E_{U}$ есть тривиальное расслоение пространства $U \times \mathbb{C}^{p}$, а $\nabla_{U}-$ связность в нем, определяемая формой $\omega$. Восстановим операторы $S_{z, z^{\prime}}$ для любых пар склеиваемых точек $z, z^{\prime} \in \partial U$. Точки $z, z^{\prime} \in \partial U$ лежат на склеиваемых ребрах $x_{0}^{i} x_{0}^{i+1}$ и $x_{0}^{j+1} x_{0}^{j}$ (порядок точек отражает тот факт, что мы склеиваем их с разной ориентацией).

ПрЕДЛОЖЕНИЕ 3. Пусть $Y_{1}$ - матрица, столбцъ которой суть горизонтальные сечения расслоения $E_{U}$ над ребром $x_{0}^{i} x_{0}^{i+1}$ с начальным условием $Y_{1}\left(x_{0}^{i}\right)=E$. Пусть $Y_{2}$ - матрица, столбиы которой суть горизонтальные сечения расслоения $E_{U}$ над ребром $x_{0}^{j+1} x_{0}^{j}$ с начальным условием $Y_{2}\left(x_{0}^{j+1}\right)=S_{x_{0}^{i} x_{0}^{j+1}}=S_{x_{0}^{1} x_{0}^{i}}^{-1} S_{x_{0}^{1} x_{0}^{j+1}}$. Тогда $S_{z, z^{\prime}}=Y_{2}\left(z^{\prime}\right) Y_{1}(z)^{-1}$.

ДокАЗАТЕЛьство. Поскольку ребра $x_{0}^{i} x_{0}^{i+1}$ и $x_{0}^{j+1} x_{0}^{j}$ склеиваются в один разрез, слои $E_{U}$ над точками из этих ребер должны склеиться в слои $E$. При этом матрицы $Y_{1}$ и $Y_{2}$ перейдут в два набора горизонтальных сечений над этим разрезом. Начальные условия для данных горизонтальных сечений совпадают, так как набор сечений $Y_{1}\left(x_{0}^{i}\right)$ расслоения $E_{U}$ над точкой $x_{0}^{i}$ отождествляется с набором сечений $S_{x_{0}^{i} x_{0}^{j+1}} Y_{1}\left(x_{0}^{i}\right)=Y_{2}\left(x_{0}^{j+1}\right)$ над точкой $x_{0}^{j+1}$. Но тогда эти два набора сечений $Y_{1}$ и $Y_{2}$ должны склеиться над всем разрезом. Из этого следует, что если точки $z \in x_{0}^{i} x_{0}^{i+1}$ и $z^{\prime} \in x_{0}^{j+1} x_{0}^{j}$ склеиваются при факторизации, то $S_{z, z^{\prime}} Y_{1}(z)=Y_{2}\left(z^{\prime}\right)$. Предложение доказано.

Тотальное пространство расслоения $E$ получается из тотального пространства $E_{U}$ следующим образом: если точки $z, z^{\prime} \in \partial U$ склеиваются при факторизации в риманову поверхность, то мы склеиваем слои $E_{U, z}$ и $E_{U, z^{\prime}}$ с помощью оператора $S_{z, z^{\prime}}$.

Связность $\nabla$ в $E$ восстанавливается автоматически. Действительно, int $U$ отображается биголоморфно на некоторое открытое всюду плотное множество $U^{\prime}$ в $M$, поэтому связность $\nabla_{U}$ однозначно определяет связность $\nabla$ в $\left.E\right|_{U^{\prime}}$. Поскольку множество $U^{\prime}$ всюду плотно и на $\partial U^{\prime}$ нет особенностей, связность однозначно определена на всей поверхности. 


\section{Подведем итог.}

ПрЕДЛОЖЕНИЕ 4. По борме $\omega$ вида (1) с особенностями в фундаментальном многоугольнике $U$ и матрицам $S_{x_{0}^{1}, x_{0}^{i}}, i=2, \ldots, 4 g$, полученным из пары $(E, \nabla)$ на поверхности, пара $(E, \nabla)$ восстанавливается следующим образом:

1) строим пару $\left(E_{U}, \nabla_{U}\right)$ - тривиальное расслоение на $U$ со связностью, определяемой формой $\omega$;

2) по правилу, описанному в предложении 3, из матрии, $S_{x_{0}^{1}, x_{0}^{i}}$ восстанавливаем матрицы $S_{z, z^{\prime}}$ для всех возможных пар точек $z, z^{\prime} \in \partial U$, склеиваемых при факторизаиии;

3) тотальное пространство $E$ получается из тотального пространства $E_{U}$ по следующему правилу: если z, $z^{\prime} \in \partial U$ склеиваются при факторизации в риманову поверхность, то мы склеиваем слои $E_{U, z} u E_{U, z^{\prime}}$ с помощью оператора $S_{z, z^{\prime}}$;

4) связность $\nabla$ в Е восстанавливается автоматически.

Выразим монодромию $\nabla$ вдоль разрезов с помощью формы $\omega$ и матриц $S_{x_{0}^{1}, x_{0}^{i}}$. Обозначим через $\left(E_{U}, \nabla_{U}\right)$ тривиальное расслоение на $U$ со связностью, определяющейся формой $\omega$.

ОПРЕДЕЛЕНИЕ 7 . Возьмем в слое $E_{U, x_{0}^{1}}$ над точкой $x_{0}^{1}$ какой-либо базис $e_{1}^{1}, \ldots, e_{p}^{1}$. Тогда в слое $E_{U, x_{0}^{i}}$ над точкой $x_{i}^{0}$ имеется базис $S_{x_{0}^{1}, x_{0}^{i}} e_{1}^{1}, \ldots, S_{x_{0}^{1}, x_{0}^{i}} e_{p}^{1}$. Такую систему базисов в слоях $E_{U, x_{0}^{i}}, i=1, \ldots, 4 g$, будем называть согласованной.

При отождествлении $E_{U, x_{0}^{i}}=E_{x^{0}}$ все эти базисы переходят в один базис $E_{x^{0}}$, который мы обозначим как $e_{1}, \ldots, e_{p}$.

Возьмем в качестве базиса слоя $E_{U, x_{0}^{1}}$ стандартный базис

$$
e_{1}^{1}=(1,0, \ldots, 0), \quad \ldots, \quad e_{p}^{1}=(0,0, \ldots, 1) .
$$

Выберем согласованную систему базисов в слоях $E_{U, x_{0}^{i}}, i=1, \ldots, 4 g$. Это даст нам базис $e_{1}, \ldots, e_{p}$ в $E_{x^{0}}$. Вычислим матрицы монодромии в этом базисе.

Лемма 1. Выпустим из точки $x_{0}^{1}$ вдоль композиции дуг $x_{0}^{1} x_{0}^{2} \ldots x_{0}^{i-1} x_{0}^{i}$ горизонтальные сечения $y_{1}, \ldots, y_{p}$ такие, что $y_{k}\left(x_{0}^{1}\right)=e_{k}^{1}, k=1, \ldots, p$, и запишем их в матрицу $Y=\left(y_{1}, \ldots, y_{p}\right)$. Тогда матрица монодромии вдоль петли, получаемой после факторизачии последовательности дуг $x_{0}^{1} x_{0}^{2} \ldots x_{0}^{i-1} x_{0}^{i}$, есть $S_{x_{0}^{1}, x_{0}^{i}}^{-1} Y\left(x_{0}^{i}\right)$.

ДокАЗАтельство. Действительно, $S_{x_{0}^{1}, x_{0}^{i}}^{-1} Y\left(x_{0}^{i}\right)$ - матрица в базисе $y_{k}\left(x_{0}^{1}\right)=e_{k}^{1}$, $k=1, \ldots, p$, оператора, который сначала горизонтально переносит сечения вдоль указанной последовательности дуг из слоя над $x_{0}^{1}$ в слой над $x_{0}^{i}$, а затем отождествляет слои, как при факторизации в расслоение $E$. По определению данная матрица есть матрица монодромии в базисе $e_{1}, \ldots, e_{p}$ вдоль петли, которая образуется после факторизации последовательности дуг $x_{0}^{1} x_{0}^{2} \ldots x_{0}^{i-1} x_{0}^{i}$. Лемма доказана.

Далее мы будем говорить "монодромия обхода по петле $x_{0}^{1} x_{0}^{2} \ldots x_{0}^{i-1} x_{0}^{i}$ ", имея в виду монодромию обхода по петле, получающейся после факторизации последовательности дуг $x_{0}^{1} x_{0}^{2} \ldots x_{0}^{i-1} x_{0}^{i}$.

Если мы знаем монодромию обхода по всем петлям $x_{0}^{1} x_{0}^{2} \ldots x_{0}^{i-1} x_{0}^{i}$, то это эквивалентно тому, что мы знаем монодромии обхода по каждому из ребер $x_{0}^{j} x_{0}^{j+1}$. Поэтому, когда в дальнейшем мы будем выписывать условие сохранения монодромии обхода по разрезам, мы будем выписывать условия сохранения монодромии обхода 
по всем петлям $x_{0}^{1} x_{0}^{2} \ldots x_{0}^{i-1} x_{0}^{i}$. Нам также понадобится, однако, выражение для монодромии обхода по каноническому разрезу, получающемуся после факторизации сторон $x_{0}^{i} x_{0}^{i+1}$ фундаментального многоугольника.

ЛЕмма 2. Матрица монодромии $\nabla$ вдоль ориентированной петли, которая получается после факторизации петли $x_{0}^{i} x_{0}^{i+1}$, записывается так: выпустим из точки $x_{0}^{i}$ вдоль $x_{0}^{i} x_{0}^{i+1}$ горизонтальные сечения $\tilde{y}_{1}, \ldots, \tilde{y}_{p}$ nарь $\left(E_{U}, \nabla_{U}\right)$ такие, что $\tilde{y}_{k}\left(x_{0}^{i}\right)=e_{k}^{i}, k=1, \ldots, p$, з запишем их в матрииу $\widetilde{Y}=\left(\tilde{y}_{1}, \ldots, \tilde{y}_{p}\right)$ (отметим, что можно написать равенство $\left.\widetilde{Y}\left(x_{0}^{i}\right)=S_{x_{0}^{1} x_{0}^{i}}\right)$. Тогда матрица монодромии вдоль петли $x_{0}^{i} x_{0}^{i+1}$ eсть $\tilde{Y}^{-1}\left(x_{0}^{i}\right) S_{x_{0}^{i}, x_{0}^{i+1}}^{-1} \tilde{Y}\left(x_{0}^{i+1}\right)=S_{x_{0}^{1} x_{0}^{i+1}}^{-1} \tilde{Y}\left(x_{0}^{i+1}\right)$.

ДокАЗАТЕЛЬСтво повторяет доказательство предыдущей леммы.

До сих пор мы исходили из того, что форма $\omega$ и матрицы $S_{x_{0}^{1}, x_{0}^{i}}$ получены из пары $(E, \nabla)$ на римановой поверхности. Теперь дадим ответ на следующий естественный вопрос. Пусть задана риманова поверхность как фактор внешности единичного диска; в диске выделен фундаментальный многоугольник. Кроме того, задана форма (1) на сфере Римана, при этом все особые точки формы, кроме, быть может, нуля, лежат в фундаментальном многоугольнике $U$, также задан набор невырожденных матриц $S_{x_{0}^{1}, x_{0}^{i}}, i=1, \ldots, 4 g$. В предложении 4 описана процедура построения расслоения со связностью на римановой поверхности по таким данным. При каких условиях ее удается осуществить?

Лемма 3. Необходимое и достаточное условие для восстановления $(E, \nabla)$ по процедуре из предложения 4 таково: пусть $x_{0}^{i} x_{0}^{i+1}$ и $x_{0}^{j+1} x_{0}^{j}$ склеиваются в один разрез. Тогда

$$
Y_{1}^{-1}\left(x_{0}^{i}\right) S_{x_{0}^{i}, x_{0}^{i+1}}^{-1} Y_{1}\left(x_{0}^{i+1}\right)=Y_{2}^{-1}\left(x_{0}^{j+1}\right) S_{x_{0}^{j+1}, x_{0}^{j}}^{-1} Y_{2}\left(x_{0}^{j}\right)
$$

где $Y_{1}$ - матрица, столбць которой суть горизонтальные сечения пары $\left(E_{U}, \nabla_{U}\right)$ вдоль дуги $x_{0}^{i} x_{0}^{i+1}$ с начальным условием $Y_{1}\left(x_{0}^{i}\right)=S_{x_{0}^{1} x_{0}^{i}}$, a $Y_{2}$ - матрица, столбць которой суть горизонтальные сечения пары $\left(E_{U}, \nabla_{U}\right)$ вдоль дуги $x_{0}^{j+1} x_{0}^{j}$ с начальным условием $Y_{2}\left(x_{0}^{j+1}\right)=S_{x_{0}^{1} x_{0}^{j+1}}$.

ДоказАтельство. Докажем необходимость. Поскольку $x_{0}^{i} x_{0}^{i+1}$ и $x_{0}^{j+1} x_{0}^{j}$ склеиваются в один разрез, монодромию обхода по этому разрезу можно вычислить как с помощью $x_{0}^{i} x_{0}^{i+1}$, так и с помощью $x_{0}^{j+1} x_{0}^{j}$, причем результат должен быть одинаковым. Монодромия, вычисленная с помощью $x_{0}^{i} x_{0}^{i+1}$, есть $Y_{1}^{-1}\left(x_{0}^{i}\right) S_{x_{0}^{i}, x_{0}^{i+1}}^{-1} Y_{1}\left(x_{0}^{i+1}\right)$, монодромия, вычисленная с помощью $x_{0}^{j+1} x_{0}^{j}$, есть $Y_{2}^{-1}\left(x_{0}^{j+1}\right) S_{x_{0}^{j+1}, x_{0}^{j}}^{-1} Y_{2}\left(x_{0}^{j}\right)$. Приравнивая эти два выражения, получаем (3).

Докажем достаточность. Всегда можно построить пару $\left(E_{U}, \nabla_{U}\right)$ - тривиальное расслоение со связностью, определяемой формой $\omega$. В силу того что $\operatorname{int} U$ отображается биголоморофно на некоторое открытое плотное множество $U^{\prime} \subset M$, над $U^{\prime}$ всегда имеется расслоение со связностью. Остается склеить его в расслоение со связностью на всей поверхности.

Возьмем точку $P=x_{0}$ и рассмотрим ее малую окрестность $O$. В этой точке $P$ сходятся все разрезы. В само́й точке $P$ склейка уже задана - ее дают матрицы $S_{x_{0}^{1}, x_{0}^{i}}$. Используя предложение 2 , можно осуществить склейку по плоским сечениям вдоль 
небольших участков разрезов, содержащихся в $O$. В результате получим расслоение со связностью $(E, \nabla)$ над этой малой окрестностью $O$, и нам остается осуществить склейку вдоль оставшихся частей разрезов, лежащих вне $О$. Мы вновь должны осуществлять склейку по горизонтальным сечениям. Оставшаяся часть разреза есть кривая, оба конца которой, $P_{1}$ и $P_{2}$, лежат на границе $\partial O$. Возьмем один из концов $P_{1}$ в качестве начальной точки и начнем процедуру склейки по горизонтальным сечениям. Нужно лишь проверить корректность процедуры склейки в другой концевой точке $P_{2}$.

Теперь мы находимся в точности в ситуации, описываемой предложением 2: в качестве $\gamma$ выступает разрез (целиком, вместе с той частью, которая лежит в $O$ ), а в качестве $V$ - малая окрестность разреза. Мы уже имеем расслоение со связностью на $V$ вне $\gamma$ и склейку по плоским сечениям на $\gamma \cap O$. Корректность слейки вдоль всей кривой $\gamma$ эквивалентна совпадению монодромии склеиваемых связностей вдоль $\gamma$. Но одно из условий (3) в точности это и выражает. Лемма доказана.

Подведем итог этого раздела.

Теорема 1. По расслоению со связностъю $(E, \nabla)$ на римановой поверхности строятся следующие данные:

1) форма (1) на сфере, при этом все особъе точки формы, кроме, быть может, нуля, лежат в фундаментальном многоугольнике $U$, а ноль - регулярная особенность;

2) набор матрии, $S_{x_{0}^{1}, x_{0}^{i}}$, где $x_{0}^{i}$ - вершины фундаментального многоугольника, $i=1, \ldots, 4 g$, при этом матрицы $S_{x_{0}^{i}, x_{0}^{i+1}}=S_{x_{0}^{1} x_{0}^{i+1}} S_{x_{0}^{1} x_{0}^{i}}^{-1}$ удовлетворяют соотношениям (3) для всех пар ребер $x_{0}^{i} x_{0}^{i+1}$ u $x_{0}^{j+1} x_{0}^{j}$, склеиваемых в один разрез. Матрииы $Y_{1}, Y_{2}$ те же, что в лемме 3 .

Верно и обратное: по таким данным можно построить расслоение со связностью на римановой поверхности.

\section{4. ИЗОМОНОДРОМНЫЕ ДЕФОРМАЦИИ}

В предыдущем разделе паре $(E, \nabla)$ на поверхности были сопоставлены форма (1) на сфере Римана с регулярной особенностью в нуле и матрицы $S_{x_{0}^{1}, x_{0}^{i}}$. Посмотрим, как задаются изомонодромные деформации пар $(E, \nabla)$ при таком соответствии.

Пусть $\left(E^{1}, \nabla^{1}\right)$ - пара на $\widetilde{T}_{1}$. Для $\tau \in \widetilde{T}$ обозначим через $\left.\left(E^{1}, \nabla^{1}\right)\right|_{\tau}$ ограничение расслоения со связностью $\left(E^{1}, \nabla^{1}\right)$ на подпространство $\left.\widetilde{T}_{1}\right|_{\tau}$ (см. определение 3 ).

ОПРЕДЕЛЕНИЕ 8. Изомонодромное семейство есть пара $\left(E^{1}, \nabla^{1}\right)$ на $\widetilde{T}_{1}$ такая, что:

1) пара $\left(E^{1}, \nabla^{1}\right)$ имеет особенности на гиперповерхностях $z=a_{i}$ (точнее, на гиперповерхностях в $\widetilde{T}_{1}$, являющихся прообразами гиперповерхностей $z=a_{i}$ в $T_{1}$ );

$2)$ для всех $\tau \in \widetilde{T}$ пары $\left.\left(E^{1}, \nabla^{1}\right)\right|_{\tau}$ имеют одну и ту же монодромию.

Пусть точка $\tau_{0} \in \widetilde{T}$. Пусть $(E, \nabla)$ - пара на отмеченной римановой поверхности, отвечающей $\tau_{0}$, причем особенности $\nabla$ соответствуют отмеченным точкам в $\tau_{0}$.

ОПРЕДЕЛЕНИЕ 9. Будем говорить, что изомонодромное семейство $\left(E^{1}, \nabla^{1}\right)$ описывает деформацию пары $(E, \nabla)$, если $\left.\left(E^{1}, \nabla^{1}\right)\right|_{\tau_{0}}=(E, \nabla)$. 
ОПредЕЛЕНиЕ 10. Семейство $\left(E^{1}, \nabla^{1}\right)$ называется шлезингеровским, если при фиксированной точке пространства Тейхмюллера в некоторой окрестности гиперповерхности $z=a_{i}$ связность $\nabla^{1}$ задается в локальных координатах формой вида

$$
\frac{B_{i}}{\zeta-a_{i}} d\left(\zeta-a_{i}\right)+h\left(\zeta, a_{i}\right)
$$

где $h\left(\zeta, a_{i}\right)$ - некоторая голоморфная форма, $B_{i}$ - голоморфная функция от $a_{i}$.

Сформулируем теперь утверждение о глобальном существовании деформаций.

ПрЕДЛОЖЕНИЕ 5. Для каждой логарифмической начальной пары $($ Е, $\nabla)$ при $t_{0} \in \widetilde{T}$ существует и единственно ее продолжсение до шлезингеровского изомонодромного семейства $\left(E^{1}, \nabla^{1}\right)$.

ДоказАтельство. Так как данное утверждение известно, дадим только набросок доказательства. Задача состоит в построении пары $\left(E^{1}, \nabla^{1}\right)$ на $\widetilde{T}_{1}$. Прежде всего, отметим, что существует изоморфизм $\pi_{1}\left(M \backslash\left\{a_{1}^{0}, \ldots, a_{n}^{0}\right\}\right) \rightarrow \pi_{1}\left(\widetilde{T}_{1}\right)$ (здесь $a_{1}^{0}, \ldots, a_{n}^{0}$ - начальные положения особых точек); это является следствием гомотопической эквивалентности. Используя конструкцию Рёрля [13], можно построить пару $\left(E^{1}, \nabla^{1}\right)$ на $\widetilde{T}_{1}$ вне малых окрестностей гиперповерхностей $z=a_{i}$. Задача построения $\left(E^{1}, \nabla^{1}\right)$ в окрестности особой гиперповерхности $z=a_{i}$ является локальной, и рассуждения в данном случае не отличаются от аналогичных рассуждений в случае сферы Римана. Таким образом получается пара $\left(E^{1}, \nabla^{1}\right)$ на $\widetilde{T}_{1}$, что и завершает доказательство.

Установим теперь связь между семейством $\left(E^{1}, \nabla^{1}\right)$ на $\widetilde{T}_{1}$ и семейством форм из теоремы 1. Рассмотрим шлезингеровское семейство $\left(E^{1}, \nabla^{1}\right)$ и точку $t^{0} \in \widetilde{T}_{1}$. Обозначим особые точки, отвечающие $t^{0}$, через $a_{1}^{0}, \ldots, a_{n}^{0}$, а точку пространства Тейхмюллера, отвечающую $t^{0},-$ через $\tau^{0}$. Пусть $W_{a_{i}^{0}}$ - достаточно малая окрестность точки $a_{i}^{0}$ (такая, что $W_{a_{i}^{0}} \cap W_{a_{j}^{0}}=\varnothing$, если $\left.i \neq j\right), i=1, \ldots, n$, а $V_{\tau^{0}}$ - достаточно малая окрестность точки $\tau^{0}$ в пространстве Тейхмюллера.

ПреДЛОЖЕНИЕ 6. Существует форма $\omega^{1}$ на пространстве

$$
\left\{\left(z, a_{1}, \ldots, a_{n}, \tau\right): z \in \overline{\mathbb{C}}, a_{i} \in W_{a_{i}^{0}}, i=1, \ldots, n, t \in V_{\tau^{0}}\right\},
$$

которая обладает следующими свойствами:

1) справедливо представление

$$
\omega^{1}=\frac{C_{k}}{z^{k}} d z+\cdots+\frac{C_{1}}{z} d z+\sum_{i=1}^{n} \frac{B_{i}}{z-a_{i}} d\left(z-a_{i}\right)
$$

2) если мы зафиксируем точку $t$, достаточно близкую $\kappa t^{0}$, рассмотрим пару $\left.\left(E^{1}, \nabla^{1}\right)\right|_{t}$ и форму $\omega$, которые отвечают паре $\left.\left(E^{1}, \nabla^{1}\right)\right|_{t}$ по теореме 1 , то форма $\omega$ может быть получена путем фиксирования особых точек в форме $\omega^{1}$ в отмеченных точках, соответствующих зафиксированной точке $t^{1)}$.

${ }^{1)}$ Важно отметить, что форма $\omega^{1}$ не содержит координат на $V_{\tau^{0}}$ и дифференциалов этих координат. 
ДокАЗАТЕЛьство. Возьмем пересечение всех канонических фундаментальных многоугольников, которые отвечают всем точкам пространства Тейхмюллера, принадлежащим $V_{\tau}$. Пусть $O$ - открытое подмножество этого пересечения, граница которого есть гладкая кривая без самопересечения. Предположим, что окрестность $V_{\tau^{0}}$ настолько мала, что $W_{a_{i}^{0}} \subset O$ для всех $i=1, \ldots, n$.

Существует отображение

$$
f: O \times W_{a_{1}^{0}} \times \cdots \times W_{a_{n}^{0}} \times V_{\tau} \rightarrow T_{1},
$$

которое определяется следующим образом. Возьмем точку

$$
\left(z, a_{1}, \ldots, a_{n}, t\right) \in O \times W_{a_{1}^{0}} \times \cdots \times W_{a_{n}^{0}} \times V_{\tau} .
$$

Используя точку $t$ в пространстве Тейхмюллера, можно восстановить подгруппу $G_{t} \subset$ Aut $D$ (напомним, $D$ есть внешность единичного диска). Обозначим как $z / G_{t}$ образ точки $z \in O \subset D$ на отмеченной римановой поверхности при факторизации $D \rightarrow M$ под действием $G_{t}$. Тогда $f$ переводит точку $\left(z, a_{1}, \ldots, a_{n}, t\right)$ в точку $\left(t, z / G_{t}, a_{1} / G_{t}, \ldots, a_{n} / G_{t}\right) \in T_{1}$. Так как образ этого отображения достаточно мал, отображение (5) существует. Отметим, что это отображение не голоморфно, но оно становится голоморфным, если мы зафиксируем точку в пространстве Тейхмюллера.

Возьмем обратный образ $\left(E_{O}^{1}, \nabla_{O}^{1}\right)$ пары $\left(E^{1}, \nabla^{1}\right)$ при отображении $f$. При фиксированной точке в пространстве Тейхмюллера этот образ есть голоморфно тривиальное расслоение со связностью.

При фиксированных параметрах $a_{1}, \ldots, a_{n}, \tau$ можно продолжить $\left(E_{O}^{1}, \nabla_{O}^{1}\right)$ с подмножества $O$ на всю сферу Римана $\overline{\mathbb{C}}$. Отметим, что связность $\nabla_{O}^{1}$ голоморфно зависит от $a_{1}, \ldots, a_{n}$, а расслоение $E_{O}^{1}$ по построению не зависит от $a_{1}, \ldots, a_{n}$. Следовательно, для фиксированного $\tau \in V_{\tau^{0}}$ голоморфная пара $\left(E_{O}^{1}, \nabla_{O}^{1}\right)$ продолжается с $O \times W_{a_{1}^{0}} \times \cdots \times W_{a_{n}^{0}} \times\{\tau\}$ до голоморфной пары на $\overline{\mathbb{C}} \times W_{a_{1}^{0}} \times \cdots \times W_{a_{n}^{0}} \times\{\tau\}$.

Согласно теореме Биркгофа-Гротендика с параметрами [1] существует мероморфная тривиализация этого расслоения, которая является голоморфной тривиализацией на $\overline{\mathbb{C}} \backslash\{0\} \times W_{a_{1}^{0}} \times \cdots \times W_{a_{n}^{0}} \times\{\tau\}$. Форма $\omega^{1}$ есть форма связности $\nabla_{O}^{1}$ в этой тривиализации. Отметим, что $\omega^{1}$ может (неголоморфно) зависеть от $\tau$. Напомним, что $\left(E^{1}, \nabla^{1}\right)$ - шлезингеровское семейство. Это сразу дает нам, что для фиксированного $\tau$ форма $\omega^{1}$ имеет вид

$$
\frac{C_{k}}{z^{k}} d z+\cdots+\frac{C_{1}}{z} d z+\sum_{i=1}^{n} \frac{B_{i}}{z-a_{i}} d\left(z-a_{i}\right)+\sum_{i=1}^{n} D_{i} d a_{i} .
$$

Выберем другую тривиализацию, в которой коэффициент при $d a_{i}$ обращается в ноль. Она строится следующим образом. Семейство $\left(E^{1}, \nabla^{1}\right)$ изомонодромно, следовательно, семейство форм $\omega^{1}$ также изомонодромно. Это эквивалентно тому, что $d \omega^{1}=\omega^{1} \wedge \omega^{1}$, т. е. форма $\omega^{1}$ интегрируема. Пусть $(p \times p)$-матрица $Y_{0}\left(z, a_{1}, \ldots, a_{n}\right)$ есть решение системы $d Y_{0}=\omega^{1} Y_{0}$ (дифференциал берется по переменным $a_{1}, \ldots, a_{n}$ ) при условии $Y_{0}\left(\infty, a_{1}^{0}, \ldots, a_{n}^{0}\right)=I$. Пусть $Y_{0}^{\infty}=Y_{0}\left(\infty, a_{1}, \ldots, a_{n}\right)$. Определим новую тривиализацию $E_{O}^{1}$ на $\overline{\mathbb{C}} \backslash\{0\} \times W_{a_{1}^{0}} \times \cdots \times W_{a_{n}^{0}} \times\{\tau\}$, подействовав на старый базис матрицей $\left(Y_{0}^{\infty}\right)^{-1}$, чтобы получить новый базис в слое. Форма связности $\nabla_{O}^{1}$ в этой новой тривиализации и есть форма $\omega^{1}$. 
По построению в новой тривиализации существует решение системы $d Y=\omega^{1} Y$ такое, что $Y\left(\infty, a_{1}, \ldots, a_{n}\right) \equiv I$ для всех $a_{1}, \ldots, a_{n}$. Так как

$$
\left.\frac{\partial Y}{\partial a_{i}}\right|_{z=\infty}=\left.\frac{B_{i}}{z-a_{i}}\right|_{z=\infty}+D_{i}=D_{i},
$$

мы имеем $D_{i}=0$. Таким образом, в новой тривиализации $E_{O}^{1}$ на пространстве $\overline{\mathbb{C}} \backslash\{0\} \times W_{a_{1}^{0}} \times \cdots \times W_{a_{n}^{0}} \times\{\tau\}$ форма $\omega^{1}$ записывается в виде (4).

Отметим, что изменение точки пространства Тейхмюллера приводит только к изменению фундаментального многоугольника. Но процедура построения тривиализации $E_{O}^{1}$ в п. 3.1 не зависела от его точной формы. Таким образом, тривиализация $E_{O}^{1}$ может быть выбрана так, что равенство (4) для $\omega^{1}$ выполняется на всем пространстве $\overline{\mathbb{C}} \backslash\{0\} \times W_{a_{1}^{0}} \times \cdots \times W_{a_{n}^{0}} \times V_{\tau}$. Предложение доказано.

Теперь выпишем уравнения изомонодромных деформаций. Первая группа уравнений описывает шлезингеровские деформации формы (1). Очевидно, что если пара $(E, \nabla)$ деформируется изомонодромно, то соответствующая форма $\omega$ также деформируется изомонодромно.

Мы рассматриваем шлезингеровские деформации, поэтому изомонодромные деформации $\omega$ задаются формой $\omega^{1}$ вида (4). Форма $\omega^{1}$ задает изомонодромную деформацию тогда и только тогда, когда $d \omega^{1}=\omega^{1} \wedge \omega^{1}$. Отметим, что $\omega^{1}$ не содержит координат на пространстве Тейхмюллера и дифференциалов этих координат, следовательно, дифференциал берется только по переменным $z, a_{i}$.

ПредЛОЖЕНИЕ 7. Уравнения изомнодромной дебормации ш имеют вид

$$
\begin{gathered}
d B_{i}=-\sum_{\substack{j=1, j \neq i}}^{n} \frac{\left[B_{i}, B_{j}\right]}{a_{i}-a_{j}} d\left(a_{i}-a_{j}\right)+\frac{\partial C_{1}}{\partial a_{i}} d a_{i}, \\
\frac{\partial C_{2}}{\partial a_{i}}-\frac{\partial C_{1}}{\partial a_{i}} a_{i}=-\left[B_{i}, C_{1}\right], \quad \ldots, \quad \frac{\partial C_{l+1}}{\partial a_{i}}-\frac{\partial C_{l}}{\partial a_{i}} a_{i}=-\left[B_{i}, C_{l}\right], \\
\ldots \ldots \ldots \ldots \ldots \ldots \ldots \ldots, \ldots \ldots \ldots, \ldots \ldots \ldots, \ldots \ldots
\end{gathered}
$$

$B$ типичном случае $C_{l}=0, l>1$, если мы положим $a_{0}=0, B_{0}=C_{1}=-\sum_{i=1}^{n} B_{i}$, то система превращается в обычную систему Шлезингера

$$
B_{i}=-\sum_{\substack{j=0, j \neq i}}^{n} \frac{\left[B_{i}, B_{j}\right]}{a_{i}-a_{j}} d\left(a_{i}-a_{j}\right) .
$$

Подчеркнем, что теперь $i=0,1, \ldots, n$.

ДокАЗАтЕЛьСтво. В явном виде равенство $d \omega^{t}=\omega^{t} \wedge \omega^{t}$ может быть переписано следующим образом. Сначала преобразуем левую часть:

$$
\begin{aligned}
\sum_{i=1}^{n} \frac{d B_{i}}{z-a_{i}} d\left(z-a_{i}\right)+\sum_{l=1}^{k} \frac{d C_{l}}{z^{l}} d z & =\sum_{i, j=1}^{n} \frac{\partial B_{i}}{\partial a_{j}} \frac{1}{z-a_{i}} d z \wedge d\left(z-a_{i}\right)- \\
- & \sum_{i, j=1}^{n} \frac{\partial B_{i}}{\partial a_{j}} \frac{1}{z-a_{i}} d\left(z-a_{j}\right) \wedge d\left(z-a_{i}\right)+\sum_{l=1}^{k} \sum_{i=1}^{n} \frac{\partial C_{l}}{\partial a_{i}} \frac{1}{z^{l}} d z \wedge d\left(z-a_{i}\right) .
\end{aligned}
$$


Теперь запишем правую часть:

$$
\begin{gathered}
\left(\sum_{i=1}^{n} \frac{B_{i}}{z-a_{i}} d\left(z-a_{i}\right)+\sum_{l=1}^{k} \frac{C_{l}}{z^{l}} d z\right) \wedge\left(\sum_{j=1}^{n} \frac{B_{j}}{z-a_{j}} d\left(z-a_{j}\right)+\sum_{l=1}^{k} \frac{C_{l}}{z^{l}} d z\right)= \\
=\sum_{\substack{i, j=1, i \neq j}}^{n} \frac{B_{i} B_{j}}{\left(a_{i}-a_{j}\right)}\left(\frac{1}{z-a_{i}}-\frac{1}{z-a_{j}}\right) d\left(z-a_{i}\right) \wedge d\left(z-a_{j}\right)+ \\
+\sum_{l=1}^{k} \sum_{i=1}^{n} \frac{\left[B_{i}, C_{l}\right]}{\left(z-a_{i}\right) z^{l}} d\left(z-a_{i}\right) \wedge d z .
\end{gathered}
$$

Формы $d\left(z-a_{i}\right) \wedge d\left(z-a_{j}\right)$ и $d z \wedge d\left(z-a_{i}\right)$ линейно независимы [1], поэтому коэффициенты при этих формах справа и слева должны совпадать. Совпадение коэффициентов при форме $d z \wedge d\left(z-a_{i}\right)$ дает уравнение

$$
\sum_{j=1}^{n} \frac{\partial B_{i}}{\partial a_{j}} \frac{1}{z-a_{i}}+\sum_{l=1}^{k} \frac{\partial C_{l}}{\partial a_{i}} \frac{1}{z^{l}}=-\sum_{l=1}^{k} \frac{1}{z^{l}} \frac{\left[B_{i}, C_{l}\right]}{z-a_{i}} .
$$

Умножив это уравнение на $z-a_{i}$, получаем

$$
\sum_{j=1}^{n} \frac{\partial B_{i}}{\partial a_{j}}+\sum_{l=1}^{k}\left(\frac{1}{z^{l-1}}-a_{i} \frac{1}{z^{l}}\right) \frac{\partial C_{l}}{\partial a_{i}}=-\sum_{l=1}^{k} \frac{1}{z^{l}}\left[B_{i}, C_{l}\right]
$$

Рассмотрим коэффициенты при степенях $1 / z$ : для $z^{0}$ получим уравнения

$$
\sum_{j=1}^{n} \frac{\partial B_{i}}{\partial a_{j}}+\frac{\partial C_{1}}{\partial a_{i}}=0
$$

для $1 / z, \ldots, 1 / z^{l}$ получим уравнения (7) и, наконец, для $1 / z^{k}$ получим уравнение (8).

Совпадение коэффициентов при формах $d\left(z-a_{j}\right) \wedge d\left(z-a_{i}\right)$ дает уравнения, не содержащие $C_{k}$, которые эквивалентны уравнениям

$$
\frac{\partial B_{i}}{\partial a_{j}}=\frac{\left[B_{i}, B_{j}\right]}{a_{i}-a_{j}}, \quad i \neq j .
$$

Эти уравнения и уравнение (10) могут быть вместе записаны как уравнение (6).

В случае $C_{2}=\cdots=C_{k}=0$ приведенные выше рассуждения совпадают с выводом уравнений Шлезингера для деформаций формы (2) (см. монографию [1]). Именно поэтому в обозначениях $a_{0}=0, B_{0}=C_{1}=-\sum_{i=1}^{n} B_{i}$ получается обычная система Шлезингера (9). Предложение доказано.

Уравнения (6)-(8) однозначно разрешимы для любых начальных условий просто потому, что они описывают изомонодромные шлезингеровские деформации связности $\nabla_{\overline{\mathbb{C}}}$ на сфере Римана.

Выпишем второй набор уравнений, которые задают изменение матриц $S_{x_{0}^{1}, x_{0}^{i}}$. В лемме 1 было доказано, что $S_{x_{0}^{1}, x_{0}^{i}}^{-1} Y\left(x_{0}^{i}\right)$ есть матрица монодромии вдоль петли, получаемой при факторизации $x_{0}^{1} x_{0}^{2} \ldots x_{0}^{i-1} x_{0}^{i}$ (здесь $Y$ такое же, как в лемме 1 ). 
Возьмем изомонодромное семейство решений $d Y=\omega^{1} Y$ такое, что при начальном положении особых точек $a_{j}$ в точке $z=\infty$ матрица $Y$ единичная (напомним, что дифференциал в этом уравнении берется по переменным $z$ и $\left.a_{j}, j=1, \ldots, n\right)$. Форма $\omega^{1}$, заданная в (4), такова, что матрица $Y$ при $z=\infty$ тождественно равна единичной. Это следует из того, что $\left.\frac{\partial Y}{\partial a_{i}}\right|_{z=\infty}=\left.\frac{B_{i}}{z-a_{i}}\right|_{z=\infty}=0$. Поэтому далее мы предполагаем, что матрица $Y$, через которую выражается монодромия согласно лемме 1 , удовлетворяет уравнению $d Y=\omega^{1} Y$.

Вернемся к условию, что монодромия вдоль всех разрезов постоянна. Оно говорит нам, что $S_{x_{0}^{1}, x_{0}^{i}}^{-1} Y\left(x_{0}^{i}\right)=$ const или, эквивалентно, $S_{x_{0}^{1}, x_{0}^{i}}=$ const $^{-1} \cdot Y\left(x_{0}^{i}\right)$. Следовательно, матрица $S_{x_{0}^{1}, x_{0}^{i}}$ удовлетворяет тому же уравнению, что и $Y\left(x_{0}^{i}\right)$, но с другими начальными данными. Другими словами, $d_{x_{0}^{i}, a_{j}} S_{x_{0}^{i} x_{0}^{i+1}}=\left.\omega^{1}\right|_{z \mapsto x_{0}^{i}} S_{x_{0}^{i} x_{0}^{i+1}}$. Здесь $\left.\omega^{1}\right|_{z \mapsto x_{0}^{i}}$ есть форма $\omega^{1}$, в которой переменная $z$ заменена на переменную $x_{0}^{i}$. В явном виде

$$
\left.\omega^{1}\right|_{z \mapsto x_{0}^{i}}=\sum_{j=1}^{n} \frac{B_{j}}{x_{0}^{i}-a_{j}} d\left(x_{0}^{i}-a_{j}\right)+\left(\frac{C_{k}}{x_{0}^{i k}}+\cdots+\frac{C_{0}}{x_{0}^{i}}\right) d x_{0}^{i} .
$$

Сформулируем основной результат настоящей работы.

Теорема 2. Для любой началъной логарифмической паръ $(E, \nabla)$ на отмеченной римановой поверхности существует единственное шлезингеровское изомонодромное семейство $\left(E^{1}, \nabla^{1}\right)$. В терминах данных из теоремы 1 илезингеровские изомонодромные деформачии локально описываются следующим образом.

1. Вериины фундаментального многоугольника изменяют свое положение в соответствии с изменением точки в пространстве Тейхмюллера.

2. Изменение коэффициентов $C_{l}, B_{i}$ формы (1) задается уравнениями из предложения 7. B случае $C_{l}=0, l>0$, в обозначениях $a_{0}=0, B_{0}=-\sum_{i=1}^{n} B_{i}$ эти уравнения есть система Шлезингера относительно $B_{i}, i=0, \ldots, n$.

3. Изменение матрии, $S_{x_{0}^{1}, x_{0}^{i}}$ описывается уравнениями $d S_{x_{0}^{1}, x_{0}^{i}}=\omega^{1} S_{x_{0}^{1}, x_{0}^{i}}$, где $\omega_{1}$ есть форма (4), в которой вместо z записана переменная $x_{0}^{i}$. Дифференциал в левой части равенства берется по переменным $a_{j}, x_{0}^{i}$.

Видно, что расслоение в паре $(E, \nabla)$, вообще говоря, изменяется при произвольных деформациях, что отличает случай высших родов от случая рода 0 , в котором естественно рассматривать связности в фиксированном тривиальном расслоении.

\section{5. СОПОСТАВЛЕНИЕ С ПОДХОДОМ КРИЧЕВЕРА}

Кратко сравним подход к изомонодромным деформациям расслоений со связностями, предложенный в настоящей работе, с подходом Кричевера [5]. Точнее, покажем, как по параметрам, используемым Кричивером, можно восстановить форму и матрицы из теоремы 1.

Ограничимся случаем, когда отмеченная риманова поверхность фиксирована. Дело в том что в работе [5] используются комплексно-аналитические координаты на пространстве Тейхмюллера, в то время как в настоящей работе на пространстве Тейхмюллера берутся вещественно-аналитические координаты $x_{0}^{i}-$ положения вершин канонического фундаментального многоугольника, и матрицы $S_{x_{0}^{1}, x_{0}^{i}}$ являются комплексно-аналитическими функциями переменных $x_{0}^{i}, i=2, \ldots, 4 g$. Таким образом, явное сопоставление между подходом Кричевера и нашим подходом в случае 
нефиксированной отмеченной римановой поверхности включает в себя построение в явном виде выражения координат вершин фундаментального многоугольника через комплексно-аналитические координаты на пространстве Тейхмюллера. Эта задача при $g>1$ наталкивается на значительные трудности (см., например, близкую к этой проблеме работу [14]).

Итак, пусть отмеченная риманова поверхность фиксирована. Пусть задано стабильное расслоение с логарифмической связностью $(E, \nabla)$, где $E$ имеет ранг $p$ и степень $p g$. В работе [5] расслоению со связностью ставятся в соответствие некоторые параметры, которые можно разделить на две группы. Параметры первой группы определяют расслоение (параметры Тюрина), а параметры второй - связность в нем (см. $\S 2$ в [5]).

Параметры Тюрина определяются следующим образом. Существует мероморфная тривиализация стабильного расслоения степени $g$ - набор голоморфных сечений $\psi_{1}, \ldots, \psi_{p}$. В слоях $E$ над всеми точками, кроме точек $\gamma_{1}, \ldots, \gamma_{m}$ (при этом обязательно $m=p g$ ), они образуют базис слоя $E$. В слоях над точками $\gamma_{1}, \ldots, \gamma_{m}$ эти сечения являются линейно зависимыми. В рассматриваемом типичном случае ранг набора сечений $\psi_{1}, \ldots, \psi_{p}$ в этих точках равен $p-1$, так что имеется единственное линейное соотношение $a_{1}^{i} \psi_{1}\left(\gamma_{i}\right)+\cdots+a_{p}^{i} \psi_{p}\left(\gamma_{i}\right)=0$. Параметры Тюрина - это набор точек $\gamma_{1}, \ldots, \gamma_{m}$ и векторы $\left(a_{1}^{i}, \ldots, a_{p}^{i}\right), i=1, \ldots, m$. В мероморфной тривиализации $\psi_{1}, \ldots, \psi_{p}$ связности $\nabla$ соответствует форма $\widetilde{\omega}$ с дополнительными особенностями в точках $\gamma_{1}, \ldots, \gamma_{m}$ и тривиальной монодромией обхода вокруг них. В работе [5] предполагается, что имеет место типичный случай, когда эти дополнительные особенности формы $\widetilde{\omega}$ являются полюсами первого порядка.

Ко второй группе относятся некоторые параметры, задающие именно связность $\nabla$. Среди них есть параметры, отвечающие за поведение формы $\widetilde{\omega}$ в окрестности точки $\gamma_{i}$ (см. $\S 2$ и лемму 2.2 в [5]). В частности, по этим данным и параметрам Тюрина сразу восстанавливается вычет формы $\widetilde{\omega}$ в точке $\gamma_{i}$. Также имеются параметры, определяющие поведение формы $\widetilde{\omega}$ в окрестности особых точек связности (см. §4 в [5]). Среди этих параметров присутствуют положения особых точек связности и сингулярные части формы $\widetilde{\omega}$ в этих окрестностях.

В настоящей работе паре $(E, \nabla)$ поставлены в соответствие форма $\omega$ и матрицы $S_{x_{0}^{1}, x_{0}^{i}}$. Отметим, что, в отличие от параметров Кричевера, нельзя утверждать, что форма $\omega$ задает связность, а матрицы $S_{x_{0}^{1}, x_{0}^{i}}-$ расслоение, так как в процедуре восстановления расслоения участвует также форма $\omega$.

Теперь, наконец, установим соответствие между заданием $(E, \nabla)$, предложенным в настоящей статье, и предложенным Кричевером. Начнем с того, что сопоставим форму и матрицы мероморфно тривиализованному расслоению. Используя тривиализацию $\left.E\right|_{U}$, задаваемую сечениями $\psi_{1}, \ldots, \psi_{p}$, получим, что форма есть просто обратный образ формы $\widetilde{\omega}$ на фундаментальном многоугольнике (его мы также будем обозначать как $\widetilde{\omega})$ и, поскольку расслоение тривиально, получим единичные матрицы $\widetilde{S_{x_{0}^{1}, x_{0}^{i}}}=I$.

Заметим, что вычеты формы $\widetilde{\omega}$ во всех ее особых точках фактически содержатся среди параметров Кричевера, так что $\widetilde{\omega}$ на фундаментальном многоугольнике также может быть сразу восстановлена по параметрам Кричевера. Опишем процедуру восстановления формы $\omega$ и матриц $S_{x_{0}^{1}, x_{0}^{i}}$ по $\widetilde{\omega}$ и $\widetilde{S_{x_{0}^{1}, x_{0}^{i}}}=I$. Чтобы восстановить $\omega$, нужно применить к $\widetilde{\omega}$ калибровочное преобразование $\Gamma$, которое должно устранить дополнительные особенности формы $\widetilde{\omega}$ в точках фундаментального многоугольника, 
соответствующих точкам $\gamma_{1}, \ldots, \gamma_{m}$ поверхности. Преобразование Г может иметь особенности лишь в этих точках, и при этом $\Gamma\left(x_{0}^{1}\right)=I$. Такое преобразование $\Gamma$ может быть найдено по вычетам формы $\widetilde{\omega}$.

Чтобы восстановить $S_{x_{0}^{1}, x_{0}^{i}}$, нужно на матрицы $\widetilde{S_{x_{0}^{1}, x_{0}^{i}}}=I$ подействовать этим же калибровочным преобразованием по правилу $\widetilde{S_{x_{0}^{1}, x_{0}^{i}}}=I \mapsto S_{x_{0}^{1}, x_{0}^{i}}=\Gamma\left(x_{0}^{i}\right)$. Действительно, $\widetilde{S_{x_{0}^{1}, x_{0}^{i}}}$ есть матрица оператора отождествления слоев, следовательно, после замены базиса с учетом определения 5 получаем матрицу $S_{x_{0}^{1}, x_{0}^{i}}=\Gamma\left(x_{0}^{i}\right) \widetilde{S_{x_{0}^{1}, x_{0}^{i}}} \Gamma\left(x_{0}^{1}\right)^{-1}$. Но две матрицы в правой части последнего равенства единичные, так что мы получаем искомое выражение.

Благодарности. Я Хочу выразить благодарность В. А. Побережному и Р. Р. Гонцову. Работа выполнена при поддержке Программы поддержки молодых ученых кандидатов наук (грант MK-4270.2011.1).

\section{Список литературы}

[1] А. А. Болибрух, Обратные задачи монодромии в аналитической теории дифберенциальных уравнений, МЦНМО, М., 2009.

[2] D. Korotkin, N. Manojlović, H. Samtleben, J. Math. Phys., 41:5 (2000), 3125-3141, arXiv: solv-int/9910010.

[3] K. Iwasaki, Pacific J. Math., 155:2 (1992), 319-340.

[4] K. Iwasaki, "Structure of the module space of SL-operators on a Riemann surface and the monodromy preserving deformation", Meeting on Ordinary Differential Equations in the Complex Domain and Special Functions (Oberwolfach, Germany, 2-8 April, 1989), Mathematisches Forschungsinstitut Oberwolfach, 1989, 6-7.

[5] I. M. Krichever, Mosc. Math. J., 2:4 (2002), 717-752, arXiv: hep-th/0112096.

[6] A. M. Levin, M. A. Olshanetsky, "Hierarchies of isomonodromic deformations and Hitchin systems", Moscow Seminar in Mathematical Physics, Amer. Math. Soc. Transl. Ser. 2, 191(43), eds. A. Yu. Morozov et al., AMS, Providence, RI, 1999, 223-262; Classical limit of the Knizhnik-Zamolodchikov-Bernard equations as hierarchy of isomonodromic deformations. Free fields approach, arXiv: hep-th/9709207.

[7] K. Okamoto, J. Fac. Sci. Univ. Tokyo, 26 (1979), 501-518.

[8] D. A. Hejhal, Acta Math., 135:1 (1975), 1-55.

[9] T. Mano, J. Math. Phys., 50:10 (2009), 103501, 21 pp.

[10] Yu. Chernyakov, A. M. Levin, M. Olshanetsky, A. Zotov, J. Phys. A, 39:39 (2006), 12083-12101, arXiv: nlin/0602043.

[11] Д. Мамфорд, "Проективные инварианты проективных структур и их применения", в кн.: Ж. Дьёдонне, Дж. Керрол, Д. Мамфорд, Геометрическая теория инвариантов, Мир, М., 1974, 259-265.

[12] Л. Альфорс, Л. Берс, Пространства римановых поверхностей и квазиконформные отображения, ИЛ, М., 1961.

[13] H. Röhrl, Bull. Amer. Math. Soc., 68 (1969), 125-166.

[14] Yu. V. Brezhnev, Mosc. Math. J., 8:2 (2008), 233-271.

Поступила в редакцию 20.04.2011, после доработки 18.08.2011 